\title{
¿ES IMPOSIBLE EL CÁLCULO ECONÓMICO EN EL SOCIALISMO? \\ CRÍTICA A LA NUEVA LECTURA AUSTRIACA*
}

Maxi Nieto Ferrández ${ }^{a}$

"DOI: https://doi.org/10.18601/01245996.v22n42.06. Recepción: 25-022019, modificación final: 21-08-2019, aceptación: 11-10-2019. Sugerencia de citación: Ferrández, M. N. (2020). ¿Es imposible el cálculo económico en el socialismo? Crítica a la nueva lectura austriaca. Revista de Economía Institucional, 22(42), 127-151.

a Doctor en Sociología. Profesor, Universidad Miguel Hernández de E1che, Alicante, España, [m.nieto@umh.es], [https://orcid.org/0000-00027369-0749]. 


\section{¿Es imposible el cálculo económico en el socialismo? Crítica a la nueva lectura austríaca}

Resumen. Este artículo evalúa la consistencia teórica y metodológica del nuevo argumento austriaco contra la posibilidad del cálculo económico en el socialismo desarrollado por la nueva generación de autores austriacos. Primero describe la evolución de la crítica austriaca al socialismo. Luego expone los fundamentos de ese nuevo argumento, tomando como referencia el libro de Huerta de Soto Socialismo, cálculo económico y función empresarial. A partir de sus ejemplos y explicaciones, así como de otros autores austriacos, muestra que dicho argumento contra el cálculo socialista se basa en razonamientos circulares o en problemas ya resueltos por las nuevas tecnologías de la información y comunicación.

Palabras clave: cálculo económico, mercados, planificación, socialismo;JEL: B53, P21

\section{Is economic calculation impossible in socialism? Criticism of the new Austrian reading}

Abstract. This article assesses the theoretical and methodological consistency of the new Austrian argument against the possibility of economic calculation in socialism as developed by a new generation of Austrian authors. First, it describes the evolution of the Austrian critique of socialism. Then, it presents the foundations of the new Austrian argument, taking as reference Huerta de Soto's book Socialismo, cálculo económico y función empresarial. From his examples and explanations, as well as from other Austrian authors, it shows this argument against calculation in socialism is based on circular reasoning or problems already solved by the new information and communication technologies.

Keywords: economic calculation, markets, planning, socialism; JEL: B53, P21

\section{O cálculo econômico é impossível no socialismo? Críticas à nova leitura austríaca}

Resumo. Este artigo avalia a consistência teórica e metodológica do novo argumento austríaco contra a possibilidade do cálculo econômico sob o socialismo desenvolvido pela nova geração de autores austríacos. Primeiro, ele descreve a evolução da crítica austríaca ao socialismo. Em seguida, ele apresenta os fundamentos deste novo argumento por referência ao livro de cálculo econômico Huerta de Soto Socialismo e função de negócios. De seus exemplos e explicações, assim como outros autores austríacos, mostra que o argumento contra o cálculo socialista é baseada em um raciocínio circular ou problemas já resolvidos por novas tecnologias de informação e comunicação.

Palavras-chave: cálculo econômico, mercados, planejamento, socialismo; JEL: B53, $\mathrm{P} 21$ 


\section{EL CÁlCULO ECONÓMICO EN EL SOCIALISMO}

耳 1 debate formal sobre el cálculo económico y la asignación racional Ede recursos en el socialismo se inició en 1920 con el artículo de von Mises "El cálculo económico en el sistema socialista", cuyas ideas luego desarrolló en El socialismo. Análisis económico y sociológico de 1922. En esos trabajos se plantea que el problema del cálculo económico consiste en economizar medios para obtener fines determinados. Es, por tanto, el problema de determinar los costos en un marco de propiedad social de los medios de producción. El argumento de von Mises contra la posibilidad del cálculo racional en una economía socialista es sencillo: puesto que no existe un mercado de medios de producción (pues son de propiedad colectiva), dichos medios carecen de precio monetario y resulta imposible calcular los costos (reducir los insumos utilizados a un denominador común mediante alguna unidad de cuenta) y, por tanto, asignar de manera eficiente los recursos a los usos deseados, eligiendo entre las innumerables combinaciones posibles de factores de producción aquellas que minimicen los costos.

Von Mises considera que en sociedades complejas con una división del trabajo desarrollada

la tarea propiamente dicha de la dirección racional de la economía [...] consiste en poner económicamente los medios al servicio de los fines. $\mathrm{Y}$ esto no es posible sin el concurso del cálculo económico (von Mises, 2009, p. 125).

A este respecto se pregunta:

¿Cómo podría saber [una comunidad socialista] si tal o cual método de producción es verdaderamente el más ventajoso? Es cuando mucho capaz de comparar la calidad y la cantidad del resultado final de la producción lista para su consumo, pero solo en casos excepcionales estará en condiciones de comparar los gastos que se necesitan para la producción. Conoce exactamente los fines que se propone o cree al menos conocerlos y debe obrar consecuentemente, es decir, debe esforzarse por alcanzar los fines que se ha propuesto con el mínimo costo (ibíd., p. 127).

Luego argumenta que

la dirección de la comunidad socialista puede fácilmente saber qué bienes necesita con mayor apremio, pero con ello solo dispone de uno de los elementos que se requieren para el cálculo económico. Le falta el segundo elemento, la evaluación de los medios de producción [...] Pero la dirección de la economía socialista no es capaz de expresar este va-

1 Simultáneamente, y en forma independiente, el sociólogo alemán Max Weber y el economista ruso Boris Brutzkus habían llegado a conclusiones similares. 
lor [el de los medios de producción] por medio de una sola unidad de precios, como lo hace la economía basada en el intercambio, que puede dar a todos los precios una expresión común por medio de la moneda (ibíd., p. 127).

Para ilustrar la imposibilidad del cálculo económico sin precios monetarios de los medios de producción pone el ejemplo de la construcción de un ferrocarril, y sostiene que para saber si el ahorro que se obtiene en el gasto de transportar mercancías es mayor que el costo de su construcción y explotación se requiere un cálculo que "solo puede efectuarse en dinero".

Por tanto, de acuerdo con von Mises, lo que está en juego en el cálculo económico es la posibilidad de emprender acciones racionales comparando los costos y beneficios de las diferentes opciones, lo cual requiere una unidad de cuenta universalmente aceptada, en la forma de moneda. Una prueba adicional de que el problema del cálculo es para él, incontrovertiblemente, el problema de determinar los costos en alguna unidad de cuenta es que critica en forma detenida las alternativas al cálculo en dinero que sugerían los partidarios del socialismo, como el cálculo directo en tiempo de trabajo (Marx) y el cálculo en especie (que propuso Neurath inspirado en la experiencia de las economías de guerra). Von Mises objeta la heterogeneidad de ambas propuestas, que haría imposible un cálculo racional, y sostiene que el uso más valioso de los recursos requiere necesariamente el cálculo económico en precios monetarios. Por ello concluye que sin precios monetarios es imposible el cálculo económico racional, y sin cálculo económico -sentencia- "el socialismo es irrealizable” (ibíd., p. 142).

La principal respuesta al reto de von Mises provino de los "socialistas de mercado" de los años veinte y treinta, entre los que se destacaron Taylor (1971) - quien no era socialista-, Dickinson (1939), Lange (1971), Durbin (1936) y Lerner (1937). Cada uno con su matiz, esos autores defendían alguna versión del método de prueba y error, simulando el funcionamiento del mercado en un marco neoclásico de equilibrio general para aproximarse a la solución matemática formulada por Barone en 1908. Este economista encontró una similitud formal entre las economías capitalista y socialista, pues ambas se podían representar mediante un sistema de ecuaciones simultáneas, de acuerdo con los modelos de equilibrio de Walras y Pareto, pero, dada su complejidad, en la práctica solo el mercado podía resolverlo.

La contrarréplica más elaborada a los socialistas de mercado fue la de Hayek en los años cuarenta, que trasladó el debate del problema original de calcular costos (con alguna unidad de cuenta) para econo- 
mizar recursos a otro más general, de coordinación social eficiente en un marco dinámico, cuya clave sería el descubrimiento, las trasmisión y el uso de la información y del conocimiento dispersos entre los diferentes agentes. Es cierto que eso ya estaba implícito en el planteamiento de von Mises, quien destacó el papel creativo del empresario en un ambiente de incertidumbre y cambios permanentes de las condiciones técnicas y las preferencias de consumo de la población, pero como ya vimos este no fue el detonante del debate en los años veinte.

La crítica de Hayek, en el fondo dirigida contra el paradigma neoclásico del equilibrio general, señala que el error fundamental de los modelos que utilizaban los socialistas de mercado para defender la posibilidad del cálculo en el socialismo es que toman como dada toda la información para formular las ecuaciones, como si fuese algo objetivo y estuviese al alcance de los agentes. Para él, en cambio, la información que se plasma en el sistema de precios es en su origen de carácter subjetivo (pues depende de la interpretación de cada persona), está dispersa entre los distintos individuos y cambia continuamente (tanto por el desarrollo técnico como por la alteración de las preferencias), y jamás está disponible para la agencia de planificación. En "El uso del conocimiento en la sociedad", Hayek expresa en forma muy precisa cuál sería -a su entender- el problema al que se enfrenta una economía socialista:

El rasgo peculiar del problema de un orden económico racional consiste, justamente, en que el conocimiento de las circunstancias que debemos utilizar nunca existe en forma concentrada o integrada sino como fragmentos dispersos de conocimiento incompleto y frecuentemente contradictorio, que los individuos poseen por separado. E1 problema económico de la sociedad no es, entonces, un mero problema de cómo asignar unos recursos "dados" -si "dados" significa dados a una mente individual que resuelve deliberadamente el problema planteado por los "datos". Se trata, más bien, del problema de cómo asegurar el mejor uso de los recursos que conocen algunos miembros de la sociedad, para fines cuya importancia relativa solo conocen esos individuos. O, en forma más breve, del problema de usar un conocimiento que no está dado a nadie en su totalidad (Hayek, 1999, pp. 333-334).

Conforme a este planteamiento, el conocimiento relevante para la organización racional de la economía es el "conocimiento de las circunstancias particulares de tiempo y lugar", un conocimiento de naturaleza subjetiva y práctica que se encuentra disperso. Por ello, para Hayek "el problema económico de la sociedad es, ante todo, el problema de la rápida adaptación a los cambios de las circunstancias particulares de tiempo y lugar" (ibíd., p. 338). Y para poder resolverlo 
las decisiones últimas deben quedar en manos de las personas familiarizadas con esas circunstancias, por cuanto conocen directamente los cambios relevantes y los recursos inmediatamente disponibles para enfrentarlos. No podemos esperar que este problema se pueda resolver trasmitiendo todo el conocimiento a una junta central que luego lo integre y emita las órdenes respectivas. Debemos resolverlo mediante alguna forma de descentralización (ibíd., p. 338).

Pese a que nunca se dio una respuesta socialista eficaz y bien elaborada al planteamiento austriaco, después de la Segunda Guerra Mundial los argumentos de Hayek contra el socialismo quedaron arrinconados, y la lectura convencional dio por buena la solución propuesta por los socialistas de mercado. Dos razones explican este hecho. En el plano teórico, la hegemonía neoclásico-keynesiana -el marco utilizado por los socialistas de mercado para llegar a su solución- relegó el enfoque austriaco a una posición marginal y extremista; se pensaba, además, que el debate se había planteado en términos puros y polarizados -mercado o plan-, cuando en realidad se trataba de buscar la combinación adecuada de ambas "instituciones". Y en el plano práctico, el otro motivo del retroceso austriaco fue el enorme desarrollo económico, industrial y científico de la Unión Soviética, que pocos años después de la devastadora invasión alemana, lideraba la carrera espacial, convertida en la segunda potencia económica mundial.

Solo en los años ochenta, en el contexto político e ideológico del despliegue del ciclo "neoliberal", una nueva generación de economistas austriacos -entre los que se destacan Kirzner $(1988,1998)$, Lavoie (1985) y, en el ámbito de la lengua castellana, Huerta de Soto- refinó los argumentos de von Mises sobre la función empresarial y de Hayek sobre el orden espontáneo y el papel del conocimiento disperso, para formular una nueva lectura del problema del cálculo en el socialismo, una visión que, tras la restauración capitalista en la antigua Unión Soviética, hoy es hegemónica. Siguiendo las apreciaciones de Hayek, esta nueva lectura considera que el debate quedó desvirtuado desde el inicio al plantearse en un marco estático neoclásico, donde la información necesaria para formular las ecuaciones del equilibrio general se toma como dada, de modo que la solución de los socialistas de mercado era irrelevante para responder al reto planteado por von Mises y Hayek, un desafío intelectual que nunca habría sido comprendido por los partidarios del socialismo.

Según la nueva lectura austriaca, el problema económico fundamental de una sociedad extensa no es asignar recursos escasos (pero conocidos) a fines dados. Este sería un problema técnico de carácter estático, es decir, de optimización matemática en un marco 
de equilibrio general, donde se supone que se dispone de toda la información necesaria (sobre fines y medios) para resolverlo. El verdadero problema económico sería, en cambio, de carácter dinámico y de coordinación social, y para resolverlo es necesario entender el funcionamiento del orden social espontáneo, o más concretamente, del proceso social entendido como un proceso creativo y competitivo basado en el descubrimiento y la trasmisión de información a partir de la acción empresarial de los individuos. La coordinación general de la sociedad exige el cálculo racional para la asignación eficiente, que minimiza costos y satisface las preferencias de consumo y las necesidades de la población. En suma, para los austriacos actuales, el problema económico fundamental es un problema "empresarial" de interacción humana, cuya clave es descubrir y movilizar la información necesaria, sobre nuevos fines y sobre los medios requeridos para alcanzarlos, clave que depende del libre ejercicio de la función empresarial de los individuos. En palabras de Huerta de Soto, según esta nueva lectura:

el socialismo es un error intelectual, pues no cabe concebir que el órgano director encargado de intervenir mediante mandatos pueda hacerse con la información que es necesaria para coordinar la sociedad, y ello por los siguientes motivos: primero, por razones de volumen (es imposible que el órgano de intervención asimile conscientemente el enorme volumen de información práctica diseminada en las mentes de los seres humanos); segundo, dado el carácter esencialmente intransferible al órgano central de la información que se necesita (por su naturaleza tácita no articulable); tercero, porque, además, no puede trasmitirse la información que aún no se haya descubierto o creado por los actores y que solo surge como resultado del libre proceso de ejercicio de la función empresarial; y cuarto, porque el ejercicio de la coacción impide que el proceso empresarial descubra y cree la información necesaria para coordinar la sociedad (Huerta de Soto, 2015, pp. 99-100).

Aunque esta es hoy la posición dominante en esa escuela, desde los años noventa otra corriente (Salerno, 1993; Hoppe, 1996 o Hülsmann, 1997) reivindica la preeminencia del aporte de von Mises a la crítica del socialismo, centrado en los problemas dinámicos de la acción evaluación empresarial en un marco de incertidumbre, frente al de Hayek, centrado en los problemas más “estáticos” de la concentración y el uso del conocimiento disperso. Tales autores consideran que Hayek desvía la atención del verdadero problema del socialismo, ya que los valores mercantiles solo se generan en mercados libres basados en la propiedad privada, pues estas instituciones son las que permiten hacer previsiones y tomar decisiones. 
No obstante, más allá de los matices, las contribuciones de von Mises y Hayek comparten un fondo común: consideran que el libre ejercicio de la función empresarial hace posible que los individuos evalúen diversos cursos de acción, $\mathrm{y}$ así generan y trasmiten un conocimiento subjetivo no centralizable. Ambos autores conciben el proceso de mercado como algo dinámico y competitivo donde los agentes no son omniscientes. Por ello, la iniciativa descentralizada de los empresarios, a través de la cual crean nueva información, evalúan y coordinan sus actos, no podría ser simulada por ningún órgano planificador. En consecuencia, la coordinación de los planes particulares de producción no se puede imponer "coactivamente" porque el centro planificador carece de la información necesaria para ello. El mercado garantiza el uso más eficiente de los recursos "para fines cuya importancia relativa solo conocen esos individuos” (Hayek, 1945). Según Huerta de Soto (2015), el aporte esencial de Hayek fue poner de manifiesto que la idea de von Mises sobre la imposibilidad del cálculo socialista solo es un caso particular del principio general de la imposibilidad lógica de la "ingeniería social", del intento de organizar una sociedad extensa en forma racional mediante la "coacción institucional" del libre ejercicio de la función empresarial.

En suma, el debate sobre la imposibilidad del socialismo pasó del problema específico inicial de determinar los costos para economizar recursos - donde la propiedad privada es una condición necesaria para la formación de precios monetarios y crear incentivos- al problema más general de la coordinación social: compatibilizar los fines o planes de los individuos, lo cual requiere movilizar el conocimiento disperso, algo que solo está al alcance del mercado por la formación espontánea de precios monetarios.

A continuación evaluamos la consistencia de la nueva formulación del teorema de la imposibilidad del cálculo y la coordinación eficiente en el socialismo. Primero exponemos el núcleo del nuevo argumento austriaco basándonos en Socialismo, cálculo económico y función empresarial de Huerta de Soto, el texto de referencia en castellano y uno de los más traducidos a otros idiomas. Después desarrollamos la crítica a ese argumento tomando como blanco los ejemplos de Huerta de Soto, así como los de Kirzner y Hayek.

\section{EL NUEVO ARGUMENTO AUSTRIACO}

Para la escuela austriaca, la esencia del proceso social, o de la interacción social, es el carácter creativo de la acción humana, que al concebir 
continuamente nuevos fines y descubrir los medios adecuados para alcanzarlos, genera y trasmite la información y el conocimiento (subjetivo, práctico y disperso) necesarios para el cálculo económico y la coordinación social (Huerta de Soto, 2015). En esta perspectiva, la acción humana se entiende como "función empresarial": "la capacidad típicamente humana para darse cuenta de cuáles son las oportunidades de ganancia que existen en el entorno" (ibíd., p. 26) y actuar para aprovecharlas. La sociedad sería un sistema "no diseñado conscientemente por nadie" o, lo que es lo mismo, un "orden espontáneo" resultante de la continua interacción de los seres humanos. Entendida como todo acto que persigue algún fin o ganancia, la función empresarial tendría dos tipos de efectos fundamentales en el proceso social:

1. Crea nueva información (de fines y medios), a partir del descubrimiento de "oportunidades de ganancia" antes desapercibidas. Esta información es de carácter subjetivo (exclusiva de cada persona), práctica (solo se crea mediante la acción empresarial), dispersa (pues está diseminada en las mentes humanas) y tácita (porque es difícil articularla). A su vez, "la creación empresarial de información implica simultáneamente [su] trasmisión [...] en el mercado" (ibíd., p. 65), pues cuando busca lograr cierto fin el agente A descubre que necesita el recurso que $B$ posee en abundancia, le trasmite la idea de que su recurso es importante. Por ello, "solo la función empresarial [puede] generar la información [necesaria] para el cálculo económico, entendido como todo juicio estimativo sobre el resultado de los diferentes cursos de acción" (ibíd., p. 26); de modo que "sin función empresarial no es posible el cálculo económico” (ibíd., p. 67).

La idea errónea de que la información es algo objetivo tiene su origen en que parte de la información subjetiva creada empresarialmente se plasma "objetivamente" en señales (precios, instituciones, normas, "firmas", etc.) que pueden ser descubiertas y subjetivamente interpretadas por muchos en el contexto de sus acciones particulares, facilitando así la creación de nuevas informaciones subjetivas más ricas y complejas. Sin embargo, y a pesar de las apariencias, la trasmisión de información social es básicamente tácita y subjetiva, es decir no expresa y articulada, y muy resumida (ibíd., p. 65).

2. Hace posible la coordinación social, pues lleva a que los actores "tiendan a ajustar o disciplinar sus comportamientos en función de las necesidades y circunstancia de los demás" (ibíd., p. 26). Esto es posible, como ya señalamos, porque todo "desajuste social" se plasma en una oportunidad de ganancia que queda latente para ser descubierta. En resumen: 
[T] odo acto empresarial descubre, coordina y elimina desajustes sociales $y$, en función de su carácter esencialmente competitivo, hace que esos desajustes, una vez descubiertos y coordinados, ya no puedan volver a ser percibidos y eliminados por ningún otro actor [...] [E1] proceso empresarial de coordinación social jamás se detiene ni agota. Esto es así porque el acto coordinador elemental [...] consiste básicamente en crear y trasmitir nueva información que por fuerza ha de modificar la percepción general de objetivos y medios de todos los actores implicados. Esto, a su vez, da lugar a la aparición sin límite de nuevos desajustes que suponen nuevas oportunidades de ganancia empresarial, y así sucesivamente, en un proceso dinámico que nunca se termina, y que constantemente hace avanzar la civilización (ibíd., p. 78).

Por ello, de

no permitirse el libre aprovechamiento de oportunidades de beneficio, no existirá el incentivo necesario para que los actores se den cuenta de las situaciones de desajuste o descoordinación social que vayan surgiendo. En suma, no se creará información, no se trasmitirá de unos agentes a otros, y los seres humanos no aprenderán a disciplinar su comportamiento en función del de sus congéneres (ibíd., p. 92).

La conclusión que se deriva de todo ello es:

el socialismo es un error intelectual porque no es teóricamente posible que el órgano encargado de ejercer la agresión institucional disponga de la información suficiente como para dar un contenido coordinador a sus mandados (ibíd., p. 95).

\section{CRÍTICA DEL NUEVO ARGUMENTO AUSTRIACO}

\section{UN RAZONAMIENTO CIRCULAR}

Acabamos de ver que para los austriacos la esencia del proceso social, o de la sociedad entendida como estructura dinámica, es la acción empresarial motivada por la búsqueda de "oportunidades de ganancias que existen en el entorno". La clave de esta concepción es la referencia a un "entorno" en el que se desenvuelve la acción humana, de cuya naturaleza social e histórica nada se dice, pero que en todo caso se caracteriza por ofrecer "oportunidades de ganancia”. Lo decisivo aquí es entender que el único "entorno" donde es posible, en forma general y sistemática, aprovechar individualmente "oportunidades de ganancia" no puede ser otro que el mercado, donde los agentes actúan libremente en tanto propietarios privados de recursos y se relacionan a través de transacciones voluntarias de compra-venta. La economía política clásica concebía el mercado como un sistema de dependencia recíproca entre particulares independientes que, para procurarse el sustento material (que ningún dispositivo institucional 
garantiza) intercambian libremente con los demás buscando su propio interés, esto es, tratando de obtener el máximo provecho de los recursos que poseen ${ }^{2}$. Lo esencial en la visión austriaca es que lo que denomina "proceso social" no es en realidad otra cosa que el "proceso de mercado". El mismo Huerta de Soto reconoce que "en un sentido amplio, coinciden los conceptos de sociedad y mercado, por lo que la definición que damos de sociedad en el texto es plenamente aplicable al mercado" (ibíd., p. 84).

A este respecto, lo primero que cabe decir es que se basa en un razonamiento circular, pues toma como premisa -la acción empresarial-aquello que debería explicar. Así se llega a concluir que la función empresarial es necesaria para la racionalidad económica, cuando la premisa del razonamiento es que actuamos en un mundo mercantil basado en el ejercicio de la función empresarial. La ciencia social debería, en cambio, explicar por qué la acción humana adopta la forma de función empresarial en una época histórica determinada, y para ello debe dar cuenta de las condiciones institucionales específicas que llevan a adoptarla.

Pero la escuela austriaca considera que la esencia del "proceso social” (abstracto y ahistórico) es la búsqueda del interés particular (las "oportunidades de ganancia"), cuando este comportamiento lejos de ser un rasgo de la naturaleza humana es una exigencia que impone el sistema mercantil. Así, toma como causa del orden mercantil lo que en realidad es su efecto, pues la acción empresarial solo es la manera específica en que deben relacionarse los individuos en un marco histórico determinado. Puesto que en el sistema mercantil nadie tiene asegurada de antemano la supervivencia, los individuos deben buscar permanentemente las mejores relaciones de cambio posibles. Y para orientar sus acciones buscan y generan nueva información, usan sus capacidades y conocimientos (habilidades, intuiciones, talentos) y hacen evaluaciones "empresariales". En este sentido, el mercado constituye un proceso de aprendizaje y descubrimiento de información para quienes participan en él.

Los autores austriacos declaran que la libre iniciativa de los individuos en el mercado (es decir, el ejercicio de la función empresarial)

2 Como muestra Marx en El capital, al marco mercantil le es inherente la existencia de una asimetría esencial en la posesión de recursos, como la que divide a la sociedad en dos grandes clases, puesto que la inmensa mayoría de la población únicamente es propietaria (como recurso productivo) de su propia capacidad laboral, que es lo único que puede aspirar a intercambiar en el mercado, lo cual le obliga a entrar en relaciones de dependencia y explotación con la minoría propietaria de los medios de producción. 
es fundamental para el funcionamiento del mercado. Esto equivale a decir que se necesita el mercado para calcular los costos y resolver los problemas de información y coordinación que genera el propio mercado. Y si la "sociedad" se identifica con el "mercado", como hace Huerta de Soto, es inevitable que el cálculo económico racional se defina por las propiedades que adopta en el sistema mercantil. Así solo se puede concluir que el cálculo racional es imposible en una economía no mercantil. Pero es una falacia argumental tratar de probar que el cálculo racional requiere el mercado (propiedad privada y formación competitiva de precios) partiendo de la premisa de que el cálculo económico se basa en el mercado. En definitiva, los austriacos toman los rasgos de un orden social específico, como lo es el orden capitalista, como condiciones generales que definen a todo orden social complejo.

La inversión de la causalidad entre comportamiento humano y reglas sociales, mediante la cual se intenta explicar lo segundo por lo primero, es una consecuencia inevitable del individualismo metodológico en que se basa la praxeología de la escuela austriaca (von Mises, 2015). Este enfoque metodológico pretende explicar las propiedades del medio social como resultado de las acciones individuales agregadas. Pero como advertimos, esta forma de razonar es claramente circular, pues lo que hace es proyectar como rasgos del comportamiento natural de los individuos -la acción maximizadora del interés particular, aprovechando oportunidades de ganancia- lo que, como ya hemos dicho, en realidad no es sino una condición de supervivencia en un marco mercantil, es decir, una exigencia de las reglas sociales dentro de las cuales los individuos actúan. La acción humana depende del "entorno", del marco institucional en el cual se desarrolla. Por eso para Marx la clave o esencia del proceso social no puede ser nunca la mera acción individual, que se desenvuelve siempre dentro de unas reglas de juego determinadas que los individuos no eligen.

Lo que define el proceso social en cada momento histórico son justamente esas reglas sociales. El sistema de relaciones sociales dentro de las cuales o a través de las cuales actúan los individuos determina la naturaleza y la lógica de funcionamiento de una sociedad determinada. Es evidente entonces que buscar "oportunidades de ganancia”, maximizar el interés personal, etc. remite a un marco social particular, capitalista, basado en la propiedad privada de los medios de producción y el intercambio mercantil. El principal efecto teórico del individualismo metodológico es la naturalización del medio social capitalista, al que considera eterno y, paradójicamente, también 
como culminación de la historia del desarrollo humano. Por ello, la escuela austriaca piensa que sin las instituciones y los procedimientos económicos que corresponden a este orden social (propiedad priva$\mathrm{da}$, precios monetarios, incentivo del beneficio, etc.) no es posible el cálculo racional.

Con base en las explicaciones y ejemplos de Huerta de Soto, Hayek y Kirzner, en los dos apartados siguientes mostramos que la función empresarial solo es necesaria para resolver los problemas de información, cálculo y coordinación social que crea el mismo proceso mercantil basado en la propiedad privada, la competencia y la búsqueda de oportunidades de ganancia. Demostraremos así que el nuevo argumento austriaco es una tautología que no impugna la posibilidad del socialismo como orden alternativo. Está fuera del alcance de este artículo, sin embargo, esbozar los procedimientos de asignación, participación y generación de información que regirían en un modelo de economía planificada que tenga en cuenta las posibilidades tecnológicas actuales (para ello remitimos a Cockshott y Cottrell, 1993, 2008; Castillo, 2018; Cockshott y Nieto, 2017). Examinamos enseguida cada una de las dos propiedades de la función empresarial que menciona Huerta de Soto.

\section{EL PROBLEMA DE LA INFORMACIÓN SUBJETIVA}

Para evaluar la importancia de la información subjetiva en la organización eficiente de una economía compleja (bien sea capitalista o socialista), primero es necesario distinguir dos tipos que los argumentos $\mathrm{y}$ ejemplos de los austriacos confunden permanentemente, y hacen imposible entender la relevancia de esta forma de información en el socialismo: 1) la información subjetiva que remite al mismo sistema capitalista, porque se crea en la interacción basada en el intercambio mercantil entre propietarios privados de recursos que ejerciendo la función empresarial buscan aprovechar "oportunidades de ganancia" en el mercado; y 2) la información subjetiva común a todo orden económico extenso, pues siempre hay que movilizar conocimiento particular, práctico y disperso para organizar la actividad productiva: destrezas, rutinas y experiencia de los trabajadores; preferencias de consumo; planes individuales de vida (elección de estudio y profesión, valores, ocio, familia, etc.), mediante el cual cada persona establece libremente sus propios fines y los medios para alcanzarlos (trabajo, ocio, consumo, ahorro...).

Se puede decir, esquemáticamente, que la corriente praxeológica de von Mises destaca el primer tipo de información, en cuanto con- 
sidera central el proceso de evaluación y prospección empresarial de los agentes en el mercado; mientras Hayek se ocupa preferentemente del segundo tipo en cuanto concibe la sociedad como un orden espontáneo que articula conocimiento disperso, práctico y tácito. E1 cuadro 1 resume las diferencias.

Cuadro 1

Dos tipos de información subjetiva en la escuela austriaca

\begin{tabular}{llll}
\hline Autor & \multicolumn{1}{c}{$\begin{array}{c}\text { Perspectiva de } \\
\text { análisis }\end{array}$} & $\begin{array}{c}\text { Tipo de información } \\
\text { relevante }\end{array}$ & \multicolumn{1}{c}{$\begin{array}{c}\text { Problema del } \\
\text { socialismo }\end{array}$} \\
\hline Von Mises & $\begin{array}{l}\text { Dinámica } \\
\text { (acción humana) }\end{array}$ & $\begin{array}{l}\text { Evaluaciones } \\
\text { empresariales }\end{array}$ & $\begin{array}{l}\text { Imposibilidad del } \\
\text { cálculo }\end{array}$ \\
\hline Hayek & $\begin{array}{l}\text { Estática } \\
\text { (orden espontáneo) }\end{array}$ & $\begin{array}{l}\text { Conocimiento } \\
\text { disperso, práctico y } \\
\text { tácito }\end{array}$ & $\begin{array}{l}\text { Imposibilidad de } \\
\text { coordinación }\end{array}$ \\
\hline
\end{tabular}

Fuente: elaboración propia.

En lo que sigue mostramos que los casos del primer tipo ("información-von Mises"), al referirse a rasgos y procesos específicos del orden capitalista, nada prueban acerca de la viabilidad o no del socialismo; en cuanto a la información del segundo tipo ("información-Hayek"), argumentamos que se puede movilizar de manera más flexible y rápida en una economía cibernéticamente planificada como la que permite la tecnología actual (big data, inteligencia artificial, internet de las cosas, supercomputadoras), y que este tipo de información no constituye un problema para el socialismo. En resumen, comprobaremos que las críticas al socialismo basadas en el primer tipo se basan en un razonamiento circular, y que las críticas asentadas en el segundo tipo apuntan a problemas superados por el desarrollo tecnológico. Para ello usaremos los ejemplos de los mismo autores austriacos acerca de cada uno de esos dos tipos de información.

\section{Información subjetiva solo necesaria en el capitalismo}

Empecemos por los ejemplos de información subjetiva del primer tipo que cita Huerta de Soto (2015). Prácticamente en cada página de su libro repite que el conocimiento necesario para el cálculo y la coordinación social es subjetivo, disperso, práctico y tácito. Pero cuando buscamos ejemplos concretos de su importancia-que no tengan que ver con las preferencias de consumo, cuyo registro no presenta ningún problema técnico en una economía socialista- encontramos muy pocos en todo el libro. La descripción más detallada que proporciona no es un ejemplo de la vida real sino una ilustración teórica 
de cómo opera la función empresarial y del tipo de conocimiento que requiere su ejercicio (ibíd., pp. 51 y ss). Su planteamiento es el siguiente: A desea alcanzar el fin X, "para ello requiere la existencia de un medio ' $R$ ' que no se encuentra a su disposición y que no sabe dónde conseguirlo” (ibíd., p. 57). Por su parte, B persigue Y, y tiene gran cantidad del recurso $\mathrm{R}$ que no considera útil o idóneo para alcanzarlo. Este es un ejemplo de desajuste o descoordinación. Aunque "en la práctica la función empresarial podrá ser ejercida por A, por B, o simultáneamente, con igual o distinta intensidad, por cualquiera de ellos" (ibíd., p. 61), es más gráfico considerar que la lleva a cabo una tercera persona, C. En esa situación, $\mathrm{C}$ advierte dicha oportunidad, actúa como intermediario y revende más caro. Si C carece de dinero, puede convencer a otro de que se lo preste. Así, en ejercicio de la función empresarial C obtiene un "beneficio puro" con la reventa.

Ante semejante exposición, lo primero que se puede decir es que cuesta imaginar un ejemplo más alejado del funcionamiento real de las economías capitalistas. El ejemplo describe más bien el mecanismo mercantilista de comprar barato para vender caro (un caso de arbitraje). Pero aun admitiendo que fuese una descripción realista, solo mostraría la forma particular en que interactúan y crean información propietarios privados de recursos en el mercado. Y pretender basarse en ella para sacar conclusiones sobre un sistema donde rigen otros principios y mecanismos de coordinación (y donde la información sobre los recursos está disponible) es completamente absurdo. No obstante, examinemos algunos de los pocos ejemplos concretos que da este autor para comprobar que todos se basan en argumentos circulares, que destacan la importancia de un tipo de información subjetiva que solo es resultado de operar en una economía mercantil.

1. Comencemos con la "contabilidad financiera y de costos" (ibíd., p. 60), de la cual nos dice que es "un conocimiento o técnica práctica que, utilizado dentro de un determinado contexto de economía de mercado, sirve como guía para la acción generalizada de los empresarios para ayudarles a conseguir sus objetivos" (ibíd., p. 60). Es decir, sería un tipo de conocimiento clave, tipo práctico y no objetivable. Pero solo es necesario, como el mismo autor reconoce, en un "contexto de economía de mercado" y, por tanto, no en cualquier otro contexto.

2. Otro ejemplo de circularidad es cuando dice que las "decisiones relativas a los factores de producción son tan complicadas, que exigen juicios estimativos que solo pueden realizarse si se incorpora [...] la información proveniente de los precios monetarios que son resultado del [...] proceso de mercado" (ibíd., p. 192). Y añade: “El corazón de 
este proceso está constituido, precisamente, por los juicios estimativos de pérdidas y beneficios que constantemente hacen los empresarios en el mercado de factores de producción”(ibíd.,p. 192). Así reconoce que estos juicios estimativos son una exigencia del "mercado de factores de producción”; de modo que si no existiese tal mercado no harían falta esos juicios estimativos de los particulares ${ }^{3}$. La importancia relativa de los factores (en términos de costos y beneficios u oportunidades de ganancia) es algo que solo conocen los individuos en la medida en que son sus propietarios y deben sacarles un rendimiento si aspiran a sobrevivir. Pero no es así cuando no hay propietarios privados y rigen otros procedimientos de valoración, deliberación y decisión para asignar los factores.

3. Otro ejemplo de información subjetiva que menciona Huerta de Soto es la estimación de los "precios futuros" (ibíd.,pp. 179-180) -que los agentes prevén para el día de mañana- supuestamente necesarios para poder realizar el cálculo económico. Pero esto solo tiene sentido para empresarios privados que compiten y deben prever escenarios, estimando los costos y beneficios de sus acciones en un contexto de incertidumbre. De nuevo, esta no es una objeción para una economía sin incertidumbre mercantil, la cual es la que exige tales prospecciones privadas.

4. Más adelante se refiere a la información sobre la escasez y el exceso de productos, y dice que puede dar lugar a respuestas muy diferentes de los actores (ibíd., p. 253). Pero, justamente, la competencia y la acumulación compulsiva del capitalismo son las que generan y exacerban a cada paso esas situaciones de escasez/exceso de bienes, y provocan desequilibrios de todo tipo (comerciales, financieros, industriales, territoriales). Y, en todo caso, los desajustes productivos ocasionales que puedan ocurrir en una economía socialista (p. ej., debido a cambios en las preferencias de los consumidores) se podrían registrar en tiempo real y corregir sin problema con la tecnología actual.

5. Unas páginas después escribe: "E1 conocimiento del empresario tiene una forma no articulada, porque más bien se trata de una 'técnica de pensamiento' cuya aplicación depende de que el actor se encuentre situado en un contexto típico de economía de mercado, $\mathrm{y}$ solo se aprende intuitivamente conforme se va ejerciendo de una manera práctica" (ibíd., p. 256). De modo que si el actor no se halla

${ }^{3}$ En una economía socialista las decisiones sobre factores de producción se tomarían mediante procedimientos centralizados y descentralizados, incluida la optimización matemática (Cockshott y Cottrell, 1993, 2008) y órganos de planificación e inversión, con ayuda de inteligencia artificial y big data (Phillips y Rozworski, 2019). 
"en un contexto típico de economía de mercado", no haría falta ese tipo de conocimiento "no articulable".

6. Apoyándose en Hayek, dice también: "si no se permite la competencia intrasectorial se hace imposible dentro de cada sector el surgimiento de la información sobre precios y costos que es necesaria para el cálculo económico" (ibíd., p. 278). Pero, ¿’en el socialismo no se puede registrar esta información? Técnicamente hoy ya es posible calcular directamente el tiempo medio de trabajo que cuesta obtener cada producto (Cockshott y Cottrell, 1993, 1997 y 2008). La competencia, a través de la cual se impone el tiempo medio de trabajo en el mercado, solo es necesaria para calcular los costos en un sistema de propiedad privada, no de propiedad social. Huerta de Soto considera que los costos "no son algo que pueda ser conocido de forma objetiva, por un órgano de planificación o por los agentes de unos monopolios sectoriales, sino que son valoraciones subjetivas estimadas en función de la capacidad empresarial de cada agente económico que toma decisiones en el mercado" (ibíd., p. 278). Sin entrar en la crítica a la teoría subjetiva del valor, de nuevo tenemos aquí un ejemplo de circularidad: los costos son valoraciones subjetivas que remiten al mercado. En consecuencia, sin mercado (propiedad privada) no habría necesidad de valoraciones subjetivas de costos, que se podrían calcular directamente en tiempo de trabajo.

7. Una apreciación que se repite en todo el libro es que “[e]n una economía de mercado la información no está dada” (ibíd., p. 296). Por supuesto, y por definición, en una economía de mercado la información relativa a qué y cómo producir está dispersa entre los productores privados. Son ellos, lógicamente, quienes tienen planes empresariales $\mathrm{y}$ hacen valoraciones subjetivas para ver qué provecho sacan de sus recursos. Pero de nuevo esto nada dice de cómo funcionarían las cosas en una economía socialista, ni siquiera si es posible o no.

8. Para rematar, sentencia: "Allí donde no exista función empresarial nunca se generará la información que solo resulta del proceso implicado por aquella" (ibíd., p. 302). Qué gran hallazgo. Es como decir que allí donde no exista deliberación ni decisiones colectivas, lo propio del socialismo, nunca se generará la información proveniente de ese proceso democrático.

Pasemos ahora a la obra clásica de Kirzner, Competencia y empresarialidad. Allí dice: "En una economía de mercado, en cualquier momento, un enorme cúmulo de ignorancia se opone a una completa coordinación de las acciones y decisiones de los numerosos participantes en el mercado”(1998, p. 229). Por tanto, solo en “una economía de 
mercado" hay falta de conocimiento y, como consecuencia, descoordinación entre las acciones de los agentes. Y son esos desajustes los que generan “oportunidades de ganancia” (ibíd.,p. 230), una particularidad de una economía de propietarios privados de recursos. El célebre "estar alerta" de Kirzner que caracterizaría la función empresarial es, por tanto, una exigencia del esquema mercantil con incertidumbre e información escasa, pero no un requerimiento para la coordinación en una economía socialista.

En suma, como hemos visto, el razonamiento circular se reduce a decir que los precios monetarios, formados como resultado del funcionamiento espontáneo del mercado, son imprescindibles para tomar decisiones... ¡ jen el mercado!

\section{Información subjetiva necesaria en el socialismo}

Examinemos ahora el otro tipo de información subjetiva y de conocimiento disperso que no surge como consecuencia del funcionamiento capitalista y que, por ello, también sería necesario articular en una economía socialista si aspira a ser eficiente. Aquí entrarían aspectos tales como la gestión práctica de los medios de producción, el registro de las preferencias de consumo, el destino de la inversión, la definición de los planes individuales y la destreza laboral. Con respecto a este tipo de información que destaca Hayek, veremos que hoy no existe ningún impedimento técnico para movilizarla en ausencia de procesos de mercado.

En su famoso artículo "El uso del conocimiento en la sociedad", Hayek sostiene que el problema de la utilización máxima del conocimiento disperso solo se puede resolver mediante un mecanismo descentralizado de toma de decisiones. Y que "el conocimiento de las circunstancias particulares de tiempo y lugar”, que sería necesario articular para una coordinación social eficiente, nunca está disponible en forma completa para la autoridad central de planificación. Pero todos sus ejemplos ya fueron superados por el desarrollo tecnológico. Dice que

conocer y poner en funcionamiento una máquina que no se usa plenamente, utilizar mejor la habilidad de una persona [...] el fletador que se gana la vida utilizando lo que de otro modo serían barcos que viajan vacíos o casi vacíos, el agente de finca raíz cuyo conocimiento se refiere casi exclusivamente a oportunidades circunstanciales o el comerciante que obtiene ganancias debidas a las diferencias locales en los precios de las mercancías, desempeñan funciones eminentemente útiles que se basan en el conocimiento especial de circunstancias fugaces desconocidas por los demás (Hayek, 1999, p. 336). 
La automatización creciente de la producción, con la industria inteligente, tiende a reducir a cero las formas de conocimiento práctico y tácito en las empresas; y aludir a ellas contra la posibilidad del socialismo es hoy injustificado.

Huerta de Soto, por su parte, da como ejemplos de "conocimiento tácito no articulable" "jugar al golf" o "montar en bicicleta" (Huerta de Soto, 2015, p. 58). Así como la decisión de "comprar una falda” para indicar que quizás ni la misma compradora "sea capaz de darnos una explicación más detallada o formalizada de por qué su elección" (ibíd., p. 60). Pero ninguno de esos ejemplos se relaciona con la actividad productiva y la coordinación económica. En qué sentido constituyen un desafío para el socialismo es una incógnita. Su ejemplo más detallado que se relaciona con la producción es el de un agricultor que, gracias a su conocimiento práctico, modifica las tareas previstas ante un cambio meteorológico. Y para negar la posibilidad de la planificación dice que es imposible trasferir esa nueva información a un "órgano director" (ibíd., p. 99). Aunque es evidente que esa información sí se puede transferir (no hay impedimento técnico alguno para ello), no sabemos qué falta haría para el buen funcionamiento de la economía o la empresa. No nos explica por qué es necesario que ese agricultor informe a alguien (y menos aún al "órgano director") cuando cambia sobre la marcha su plan del día.

Pasemos ahora a "El cálculo socialista: la solución competitiva", de Hayek. Para criticar el modelo de ensayo y error de Lange (1971), porque toma la información como algo dado, Hayek advierte que muchos bienes de capital solo se producen "por encargo" y no tienen un precio estandarizado en el mercado (1998, p. 150). Esta certera apreciación es en realidad una enmienda sorprendente a su propia tesis, pues reconoce que muchas máquinas "rara vez se producen para un mercado, más bien se hacen bajo un contrato especial (ibíd)”. ¿Por qué entonces serían necesarios los procesos de mercado? Huerta de Soto repite eso mismo (ibíd., p. 328) y pone como ejemplo de fabricación "por encargo" la de naves industriales, buques, astilleros o bienes inmuebles. Pero si algo demuestra eso es que no son imprescindibles la competencia ni los precios de mercado para of recer y obtener esos medios de producción. ¿Por qué en una economía planificada no se podría generalizar esta forma de crear y trasmitir información no basada en la competencia mercantil?

En resumen, la tecnología actual reduce significativamente la importancia del conocimiento subjetivo (como sucede con el saber práctico, gracias a la automatización y las fábricas inteligentes) o lo 
permiten articular en forma más rápida y flexible que el rudimentario mecanismo del mercado. En cualquier caso, conviene hacer dos precisiones importantes: 1) en el capitalismo ya se centralizan la información y el conocimiento disperso, en empresas y en instituciones de todo tipo, como reconocen algunos austriacos (Hoppe, 1996); y 2) en una economía socialista no se trataría tanto de centralizar el conocimiento disperso como de movilizarlo y articularlo; una economía planificada funcionaria como un sistema distribuido, capaz de regular conscientemente sus principales flujos económicos y de información; y al tiempo de alentar la iniciativa descentralizada (en diseño industrial, en nuevos proyectos empresariales; Devine, 2002).

\section{EL PROBLEMA DE LA COORDINACIÓN SOCIAL}

La otra propiedad fundamental de la función empresarial sería su carácter coordinador del comportamiento humano, pues el incentivo de la ganancia corrige los desajustes de la economía. Para averiguar si la función empresarial es una condición necesaria para la coordinación social eficiente, primero debemos aclarar qué tipo de desajustes supuestamente corrige ${ }^{4}$. Igual que con la información subjetiva, aquí debemos distinguir dos tipos de desequilibrios de naturaleza muy distinta que también confunden los ejemplos y argumentos austriacos: 1) los que genera el mercado con su funcionamiento espontáneo y que, por tanto, estarían ausentes en el socialismo (y que por ello no requerirían la función empresarial); y 2) los que responden a causas no mercantiles, que también serán inevitables en el socialismo, pero que pueden ser resueltos técnicamente sin necesidad de la iniciativa empresarial privada.

\section{Desajustes mercantiles}

Huerta de Soto menciona distintos desajustes que la función empresarial puede corregir pero que en realidad son causados por la dispersión de recursos y el proceso atomizado de toma decisiones, es decir, causados por el funcionamiento del mercado. Por ejemplo, al hablar del cálculo monetario dice que "gracias a la función empresarial" se pueden eliminar "los desajustes que existan en la estructura productiva estableciéndose así la tendencia de coordinación que hace posible la vida social" (Huerta, 2015, p. 192). Pero los desajustes de la

4 "Supuestamente" porque no aludimos aquí a la ineficiencia, derroche y lentitud de la iniciativa privada mercantil para corregir los desequilibrios que ella misma genera (por ejemplo con las burbujas inmobiliarias). 
estructura productiva, con su tendencia intrínseca a la sobreinversión y la sobreproducción, surgen y se exacerban justamente por decisiones privadas, que tienen lugar en un entorno marcado por la opacidad y la incertidumbre.

Además, dice que la debilidad de los "modelos planométricos"-los modelos de optimización matemática- "radica en la tremenda minimización y trivialización del problema que suponen los constantes cambios de mercado" (ibíd., p. 259). De nuevo, esto podría ser válido para los modelos de socialismo de mercado de matriz neoclásica (Dickinson, Lerner) pero no para una economía socialista cibernéticamente planificada sin procesos de mercado (Cockshott y Cottrell, 1993; Castillo, 2018). Un aparato productivo socializado traduciría los cambios en la demanda final de bienes de consumo en alteraciones de los requerimientos técnico-materiales de toda la cadena de interdependencias industriales.

Kirzner, por su parte, cuando estudia el proceso coordinador del mercado pone el ejemplo de la leche $(1998$, p. 232) y dice que la prueba de técnicas se hace por ensayo y error. Pero como esas pruebas se hacen en medio de la opacidad general, se acentúan los desajustes productivos que genera la misma iniciativa empresarial privada. En el socialismo las pruebas de técnicas deben hacerse en condiciones de información transparente y más completa, sin decisiones paralelas de las empresas. Sobre la coordinación dice:

E1 proceso empresarial-competitivo se nos presenta ahora no simplemente como generador de una tendencia al equilibrio, sino como descubridor y corrector de planes y decisiones individuales discordantes. Podemos observar esto en la estructura de mercado más simple como en la más compleja (ibíd., p. 232).

Obviamente esto es así solo cuando esos "planes y decisiones individuales" se definen y realizan a partir de la posesión privada de recursos (por supuesto, de una minoría de la población).

En suma, lo único que describen todos estos ejemplos es el funcionamiento de una estructura atomizada de producción, y la forma en que opera la tendencia al equilibrio que le es inherente, mediante el aprendizaje y el reajuste permanente de los planes empresariales privados. Por consiguiente, nada de lo que describen supone problemas para una economía planificada.

\section{Desajustes no mercantiles}

Más allá de los desajustes intrínsecos al funcionamiento capitalista, en toda economía compleja son inevitables otros tipos de desequilibrios. 
Estos pueden surgir debido al cambio técnico continuo, a la modificación de las preferencias de consumo o a errores en las decisiones de inversión (que solo se revelan a posteriori, cuando se confrontan con la demanda), así como a choques externos e impredecibles como las catástrofes naturales. La cuestión aquí es que no existe ningún impedimento tecnológico para que una economía planificada capte esos desajustes y los resuelva rápidamente; y que tampoco existe ninguna traba formal que impida canalizar la iniciativa creadora de los individuos de manera descentralizada.

Uno de los ejemplos que da Huerta de Soto es la variación de inventarios, de la cual dice que el exceso y la escasez son apreciaciones subjetivas que remiten a los marcos temporales variables que considera el actor (2015, p. 327). En el socialismo cualquier entidad que tenga a su cargo la gestión de recursos (la gerencia de una empresa, un consejo de inversión o una autoridad pública) también podría tomar decisiones con arreglo a criterios muy variados. En todo caso, la escasez relativa de bienes y servicios puede registrarse en tiempo real, así como hoy gestionan la cadena de suministros empresas como Amazon o Wal-Mart (Phillips y Rozworski, 2019).

En cuanto al problema de la coordinación social, quizás el aspecto más relevante del argumento austriaco sea su apreciación, del todo infundada, de que la planificación implica coacción e incluso la supresión de los planes de vida individuales. Y ello porque, supuestamente, la planificación exigiría consensos absolutos que se habrían de imponer. Por ejemplo, Hayek considera que "para planificar a gran escala, se requiere un acuerdo entre los miembros de la sociedad acerca de la importancia de las diversas necesidades mucho más amplio que el que existe normalmente [...] y habrá que imponer una escala común de valores por medio de la fuerza y la propaganda" (1998, pp. 166-167). Esto es absurdo. En el socialismo, cada cual determina sus preferencias de consumo y su plan de vida, mientras que las decisiones que afectan al colectivo se toman democráticamente, no por imposición de una minoría propietaria. En el capitalismo, los agentes privados tratan de captar, por ensayo y error, las necesidades de los individuos. En una economía planificada puede haber una pluralidad de centros y órganos decisorios para organizar la producción, y también por ensayo y error pueden corregir los posibles desajustes, quizá mucho menores debido a que no se tomarían decisiones paralelas ni existiría incertidumbre empresarial.

En "La libertad y el sistema económico", Hayek dice que planificar "presupone un acuerdo mucho más completo acerca de la importan- 
cia relativa de los diferentes fines del que actualmente existe" (1998, p. 219). ¿Por qué ha de ser así?, ¿a qué fines se refiere? Un aparato socializado solo significa que no hay atomización productiva y que el proceso económico se puede regular mediante diversas instancias de decisión a distintos niveles.

Para los austriacos, la única coacción sobre los individuos es la que ejerce el poder público. Sin embargo, trabajar bajo órdenes ajenas en un marco de propiedad social sería tan "coactivo" como trabajar en una empresa privada. ¿Creen acaso que en el capitalismo está más coaccionado un funcionario público que un trabajador precario del sector privado?, ¿quién aprovecha mejor su talento y sus capacidades?, ¿quién puede hacer planes de vida más completos, libres y satisfactorios?

Kirzner, por su parte, sostiene que

el proceso equilibrador [...] está regido por el descubrimiento empresarial de la información -sobre nuevas fuentes de recursos, nuevas oportunidades tecnológicas, nuevas combinaciones posibles de especificaciones de los productos, nuevos tipos de preferencias del consumidor- la cual origina planes empresariales de producción que alteran los precios de los recursos y las cantidades producidas de los distintos tipos y calidades de productos (Kirzner, 1998, p. 234).

Pero, como hemos advertido, esa información se puede captar perfectamente en un marco de propiedad social mediante las nuevas tecnologías de la información y la comunicación.

\section{CONCLUSIONES}

E1 nuevo argumento austriaco contra el socialismo dice que el libre ejercicio de la función empresarial es una condición necesaria para la racionalidad económica, debido a su capacidad para crear nueva información y coordinar la sociedad. Para evaluar la consistencia de este argumento examinamos los ejemplos y descripciones de los economistas austriacos sobre esas dos propiedades de la función empresarial. Sobre la primera-capacidad para crear información subjetiva-, demostramos a partir de sus propios ejemplos que esa información solo es necesaria en el mercado o que podría movilizarse sin ningún problema en una economía planificada mediante la tecnología actual. Sobre la segunda-capacidad coordinadora-, mostramos que la función empresarial solo corrige los desajustes que genera el mercado. Vimos también que los desajustes inevitables en una economía socialista se pueden captar y corregir sin ningún problema técnico. La conclusión es entonces que no se necesita la función empresarial para el cálculo racional y la coordinación eficiente de una economía compleja. 
Vimos, además, que en el argumento austriaco contra el socialismo hay dos vertientes, asociadas a los dos principales teóricos de esa escuela, von Mises y Hayek: 1) E1 aspecto “dinámico" de la crítica (von Mises) señala los supuestos problemas de cálculo y de coordinación que resolvería la función empresarial, es decir, de las evaluaciones y previsiones de los agentes en un marco de incertidumbre. Pero, como demostramos, la función empresarial solo es necesaria en el orden mercantil. Por ello, el argumento contra el socialismo se basa en un razonamiento circular, inevitable cuando se parte del individualismo metodológico, que presupone el mercado como marco de interacción entre los agentes. 2) El aspecto "estático" de la crítica (Hayek) se centra en las propiedades del orden espontáneo y se basa en su capacidad para articular el conocimiento disperso y práctico. A este respecto vimos que la posibilidad de movilizar información o conocimiento disperso es un problema superado tecnológicamente. De modo que el intento austriaco de probar que el socialismo es lógicamente imposible resulta fallido.

\section{REFERENCIAS BIBLIOGRÁFICAS}

Adaman, F. y Devine, P. (1996). The economic calculation debate: Lessons for socialists. Cambridge Journal of Economics, 20(5), 523-537.

Barone, E. (1998). El ministro de la producción en un Estado colectivista. En J. Segura y C. Rodríguez (comps.), La economía en sus textos (pp. 289-338). Madrid: Taurus.

Castillo, V. (2018). Crítica de la razón austriaca: posibilidad e imposibilidad del socialismo (TFG). Barcelona: UB y UPC.

Cockshott, P. y Cottrell A. (1993). Towards a New Socialism. Nottingham: Spokeman.

Cockshott, P. y Cottrell A. (1997). Labour-time versus alternative value bases: A research note. Cambridge Journal of Economics, 21(4), 545-549.

Cockshott, P. y Cottrell A. (2008). Computadores y democracia económica. Revista de Economía Institucional, 10(19), 161-205.

Cockshott, P., Cottrell, A. y Michaelson, G. (2007). Cantor diagonalisation and Planning. International Journal of Unconventional Computing, 5(3), 223-236.

Cockshott, P. y Nieto, M. (2017). Ciber-comunismo. Planificación económica, computadoras y democracia. Madrid: Trotta.

Cockshott, P. y Zacharia, D. (2012). Arguments for socialism, [http://www. dcs.gla.ac.uk/ wpc/].

Devine, P. (2002). A reconsideration of the theory of entrepreneurship: a participatory approach. Review of Political Economy, 14(3), 329-355.

Dickinson, H. D. (1939). Economics of Socialism. Oxford: Oxford University Press.

Durbin, E. F. (1936). Economic calculus in a planned economy. Economic Journal, 46(158), 676-690. 
Hayek, F. A. (1998). E1 cálculo socialista: la solución competitiva [1940]. Obras Completas de F.A. Hayek, Socialismo y guerra, v. 10. Madrid: Unión Editorial.

Hayek, F. A. (1999). E1 uso del conocimiento en la sociedad [1945]. Cuadernos de Economía, 18(30), 331-345.

Hayek, F. A. (2007). Camino de servidumbre. Madrid: Alianza.

Hayek, F. A. (2010). La fatal arrogancia. Los errores del socialismo. Madrid: Unión Editorial.

Hoppe, H. H. (1996). Socialism: A property or knowledge problem? Review of Austrian Economics, 9(1), 143-149.

Huerta de Soto, J. (2000). La escuela austriaca. Mercado y creatividad empresarial. Madrid: Síntesis.

Huerta de Soto, J. (2012). La esencia de la escuela austriaca y su concepto de eficiencia dinámica. Información Comercial Española, 865, 55-70.

Huerta de Soto, J. (2015). Socialismo, cálculo económico y función empresarial. Madrid: Unión Editorial.

Hülsmann, J. G. (1997). Knowledge, judgments, and the use of property. Review of Austrian Economics, 10(1), 23-48.

Kirzner, I. (1988). The economic calculation debate: Lessons for Austrians. Review of Austrian Economics, 2, 1-18.

Kirzner, I. (1998). Competencia y empresarialidad. Madrid: Unión Editorial.

Lange, O. (1971). Sobre la teoría económica del socialismo. En O. Lange y F. Taylor (eds.), Sobre la teoría económica del socialismo (pp. 59-60). Barcelona: Ariel.

Lavoie, D. (1985). Rivalry and central planning. The socialist calculation debate reconsidered. Cambridge: Cambridge University Press.

Lerner, A. P. (1937). Static and dynamic in socialist economies. Economic Journal, 47(186), 253-270.

Mises, L. von (1920). El cálculo económico en el sistema socialista, [http:// www.hacer.org/pdf/rev10_vonmises.pdf].

Mises, L. von (2009). El socialismo. Análisis económico y sociológico. Madrid: Unión Editorial.

Mises, L. von (2015). La acción humana. Madrid: Unión Editorial.

O'Neill, J. (2006). Knowledge, planning and markets: A missing chapter in the socialist calculation debate. Economics and Philosophy, 22(1), 55-78.

Phillips, L. y Rozworski, M. (2019). People's Republic of Walmart. Londres: Verso.

Salerno, J. T. (1993). Mises and Hayek dehomogenized. Review of Austrian Economics, 6(2), 113-146.

Stalebrink, O. J. (2004). The Hayek and Mises controversy: Bridging differences. Quarterly Journal of Austrian Economics, 7(1), 27-38.

Taylor, F. (1971). La orientación de la producción en un estado socialista. En O. Lange y F. Taylor (ed.), Sobre la teoría económica del socialismo (pp. 43-58). Barcelona: Ariel. 